\title{
Primary intra-fourth ventricular meningioma: Report two cases
}

\author{
Nishanth Sadashiva, Shilpa Rao ${ }^{1}$, Dwarakanath Srinivas, Dhaval Shukla \\ Departments of Neurosurgery and ${ }^{1}$ Neuropathology, National Institute of Mental Health and Neurosciences, Bengaluru, Karnataka, India
}

\section{ABSTRACT}

Meningioma's occurring intraventricular region are rare and these occurring in the fourth ventricle is even rare. Because of the rarity, it is not usually considered as a differential diagnosis in any age group. Clinical features and Imaging is not characteristic, and most of them are thought to be some different tumor. Here, we discuss two cases harboring a primary fourth ventricular meningioma Grade II, which was surgically excised successfully. Total excision was achieved in both cases and as the tumor was firm to soft and vermian splitting was not required. Understanding the clinical features and a careful preoperative radiological examination is required to differentiate this tumor from more commonly occurring lesions at this location.

Key words: Clear cell meningioma, fibroblastic meningioma, fourth ventricular meningioma, intraventricular meningioma, telovelar approach

\section{Introduction}

Meningiomas account for $13-20 \%$ of all intracranial tumors and of these $0.5-3 \%$ occur in intraventricular location. Of the intraventricular meningiomas, only $6 \%$ are primarily fourth ventricular. ${ }^{[1]}$ About few such sporadic cases have been reported in the literature. ${ }^{[2]}$ They arise from the choroid plexus and mostly lie strictly within the ventricular cavity. Clinical features and imaging are not characteristic to identify these lesions preoperatively, and most of them are thought to be some different tumor. The importance of preoperative diagnosis lies in predicting the consistency and vascularity of tumor, which needs to be removed through narrow corridors to the fourth ventricle. Here, we discuss two cases harboring a primary fourth ventricular meningioma, which was surgically excised successfully.

\section{Address for correspondence:}

Dr. Dhaval Shukla, Department of Neurosurgery,

National Institute of Mental Health and Neurosciences,

Bengaluru - 560 029, Karnataka, India.

E-mail: neurodhaval@rediffmail.com

\begin{tabular}{|l|l|}
\hline \multicolumn{2}{|c|}{ Access this article online } \\
\hline Quick Response Code: & Website: \\
\hline & www.ruralneuropractice.com \\
\hline & \\
\hline
\end{tabular}

\section{Case Reports}

\section{Case 1}

A 50-year-old male had a headache and swaying while walking since 8 months and had developed diplopia on looking to the right side. On examination, he had ataxia, right sixth nerve paresis, and papilledema. Computerized tomography (CT) scan showed an iso-hyperdense mass lesion in the fourth ventricle which was heterogeneously enhancing on contrast. Magnetic resonance imaging (MRI) showed a T1 and T2 isointense heterogeneously enhancing mass lesion with areas of nonenhancing cavities. The fourth ventricular floor appeared to be free from the lesion [Figure 1a-d]. With this features the possibilities of metastasis, ependymoma, and choroid plexus tumor was considered.

The patient underwent midline suboccipital craniotomy, telovelar approach, and excision. Lesion was located in the fourth ventricle toward the right displacing the vermis to left, entirely intraventricular, attached to

This is an open access article distributed under the terms of the Creative Commons Attribution-NonCommercial-ShareAlike 3.0 License, which allows others to remix, tweak, and build upon the work non-commercially, as long as the author is credited and the new creations are licensed under the identical terms.

For reprints contact: reprints@medknow.com

How to cite this article: Sadashiva N, Rao S, Srinivas D, Shukla D. Primary intra-fourth ventricular meningioma: Report two cases. J Neurosci Rural Pract 2016;7:276-8. 


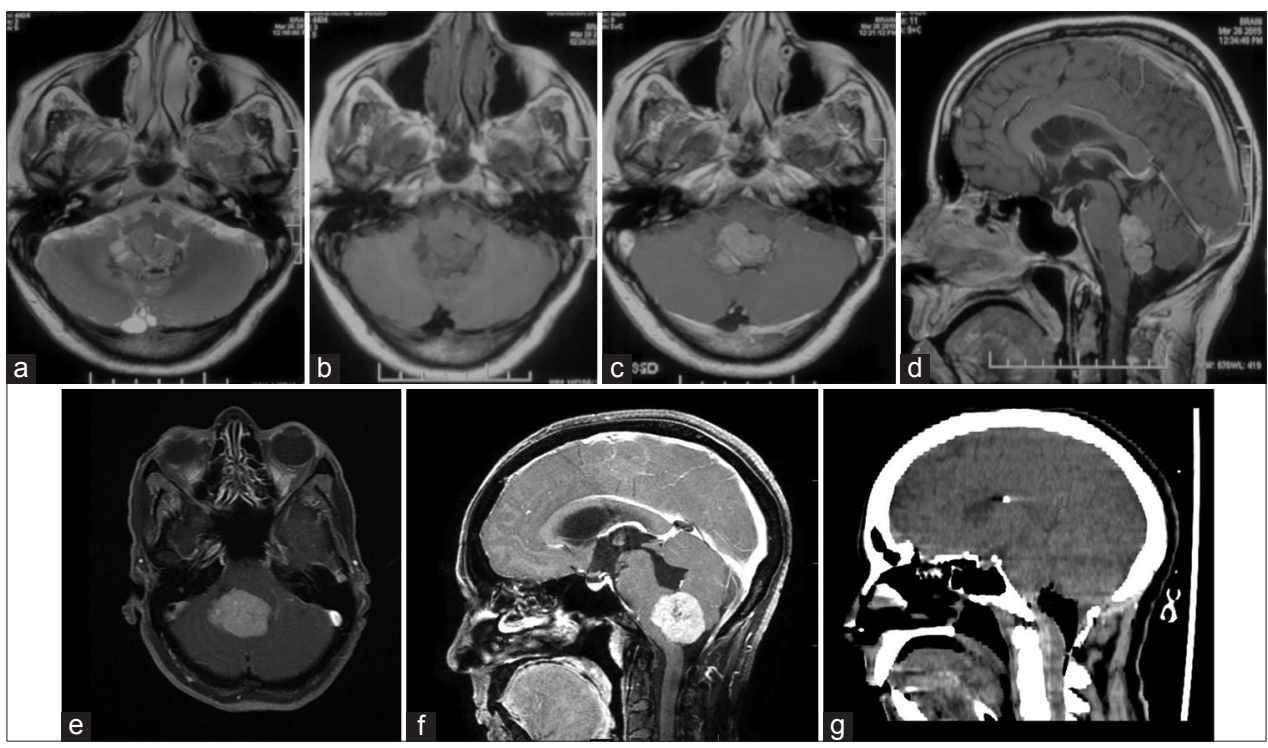

Figure 1: (a) T2-weighted image showing a heterogenous isointense intra fourth ventricular mass, (b) the lesion is isointense on T1-weighted imaging, (c and d) the lesion is enhancing on contrast administration, and it has multiple lobulations and is entirely inside the fourth ventricle. (e and f) contrast enhanced axial magnetic resonance imaging of and sagittal computed tomography, respectively of case 2 and ( $g$ ) a postoperative computed tomography showing complete excision

the choroid plexus, and posterior inferior cerebellar artery feeders were seen, during surgery supplying the lesion, which was coagulated and removed. Gross total excision was achieved. Postoperatively patient had no neurological deficits and imaging showed complete excision of tumor. Histopathological examination of the lesion led to the diagnosis Fibroblastic meningioma with brain invasion, WHO Grade II [Figure 2]. MIB-1 was $12 \%$. Patient is asymptomatic at follow-up of 6 months.

\section{Case 2}

A 47-year-old female presented with a headache and neck pain of 1-year duration along with gait disturbance and recurrent falls of 4-month duration. On examination, she had features suggestive of papilledema. She also had an ataxic gait and a wide stance suggestive of cerebellar involvement. MRI of the brain showed a predominantly intra fourth ventricular mass with extension into the cerebellomedullary cistern. It was isointense on T1, iso to hyperintense on $\mathrm{T} 2$ with homogenous contrast enhancement. There was no clear, identifiable plane between the lesion and fourth ventricular floor in MRI [Figure 1e and f]. In view, that the lesion was located a bit laterally and easily approachable through cerebellopontine angle cistern she underwent a right retromastoid suboccipital craniotomy, $\mathrm{C} 1$ arch excision and gross total excision of the meningioma. There was no dural attachment noted during surgery. The patient made an uneventful recovery. Postoperative computed tomogram of the head showed complete excision [Figure $1 \mathrm{~g}$ ], and the patients are asymptomatic at follow-up of 9 months.

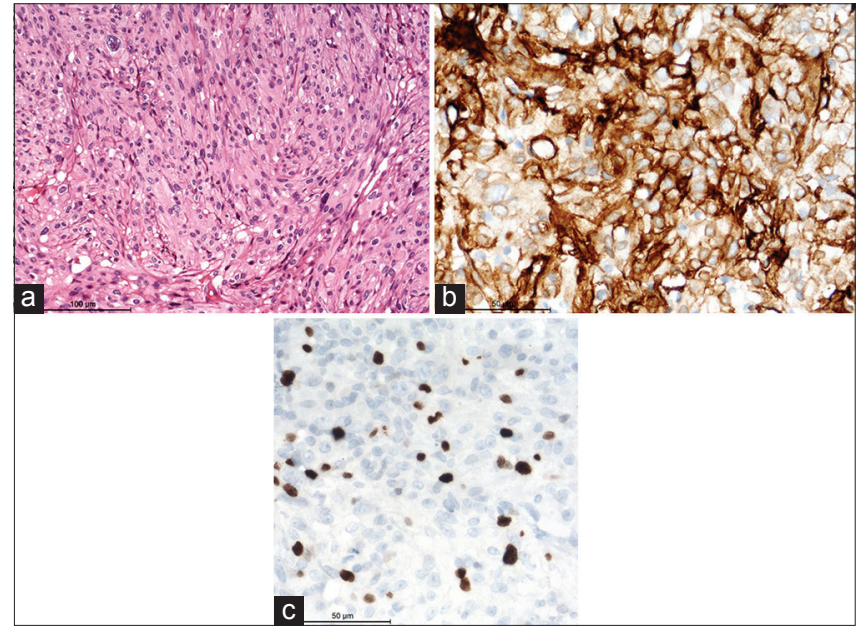

Figure 2: Photomicrograph showing interlacing fascicles of meningothelial cells on hematoxylin-eosin staining (a), expression of epithelial membrane antigen (b), and high MIB-1 labeling (c), on immunoperoxidase (magnification $=$ scale bar)

\section{Discussion}

Meningiomas arising in the ventricular system without dural attachment is extremely rare, with an incidence of $0.5-3 \%$ among all intracranial meningiomas ${ }^{[1]}$ and still rare are these located in the fourth ventricle. Fourth ventricle meningioma (FVM) was first described and surgically removed by Sachs in 1983. ${ }^{[1]}$ Since then 50 FVM have been reported in different individual reports. ${ }^{[2]}$ It appears that it is more common in females and occur at the average age of 45 years (range: $14-71$ years). ${ }^{[3]}$ They can present with a wide variety of symptoms like intracranial hypertension related to obstructive hydrocephalus, 
cerebellar dysfunction, focal neurological deficits such as diplopia, facial nerve paralysis, long tract signs, and even fluctuating symptoms due to the intermittent occlusion of the fourth ventricle. ${ }^{[2]}$

FVM is unlikely to be considered in differential diagnosis of fourth ventricular tumors in adults because it has no characteristic imaging features on CT scan or MRI. Metastasis, pilocytic astrocytoma, medulloblastoma, ependymoma, and choroid plexus papilloma are usually considered as differential diagnosis. Features such as heterogeneous enhancement, anatomical detail, ill-defined margin, and tumor extent are clues for diagnosis of meningioma in this location. Malignant tumors show lower apparent diffusion coefficient (ADC) values; on the other hand, the ADC values of pilocytic astrocytoma, hemangioblastoma, and ependymoma are higher. ${ }^{[2]}$

Meningiomas in posterior fossa without dural attachment have been classified as Type I: Meningiomas that arise from the choroidal plexus of the fourth ventricle and located entirely inside ventricular cavity, Type II: Meningiomas that arise from the inferior tela choroidea and partially located cerebellar hemisphere, and Type III: Meningiomas located within the cisterna magna but not implanted in the dura mater. ${ }^{[1]}$ According to this classification, our first case is of Type I, and second is Type II.

Resection of fourth ventricular meningioma is surgically challenging. Although almost all reported cases have been totally resected, the technical nuances may cause a potential problem due to the consistency and location. As these tumors may be firm and fibrous depending on the type of meningioma, resection may cause bleeding, and difficulty maneuvering in the narrow operative corridor, and may need vermian splitting in few cases. Intraoperative neurophysiological monitoring is an important and adjunct helps to prevent operative injury of cranial nerves and the brainstem. In our cases, the lesion was moderately firm, and total excision could be achieved. Perioperative cerebrospinal fluid diversion would be necessary only in cases with severe hydrocephalus or to tide over severe raised intracranial pressure if any, preoperatively.

The majority of the reports are Grade I meningioma's, four cases are reported to be Grade $\mathrm{II}^{[4-6]}$ and there are no reports of recurrences. However, if there is residue in case of higher grade meningiomas, then radiotherapy may have to be considered. ${ }^{[7]}$ Both our reported cases are Grade I. Like any other meningioma cases, we recommend follow-up based on the histopathological grade and extent of resection, closer follow-up will be required in higher grade meningiomas, especially if the lesion is incompletely excised.

\section{Conclusion}

Meningiomas which occur primarily in the fourth ventricle are very rare and this only the second report from India. Total removal of the lesion with good microsurgical technique is the standard and has a good outcome with low operative risk. Understanding the clinical features and a careful preoperative radiological examination is required to differentiate this tumor from more commonly occurring lesions at this location. As more and more such cases are being reported the differential diagnosis of meningioma should also be kept in mind for lesions in this location.

\section{Financial support and sponsorship}

Nil.

\section{Conflicts of interest}

There are no conflicts of interest.

\section{References}

1. Alver I, Abuzayed B, Kafadar AM, Muhammedrezai S, Sanus GZ, Akar Z. Primary fourth ventricular meningioma: Case report and review of the literature. Turk Neurosurg 2011;21:249-53.

2. Qin Y, Kanasaki Y, Takasugi M, Shinohara Y, Kaminou T, Kurosaki M, et al. Primary fourth ventricular meningioma: A case report of an adult male. Clin Imaging 2012;36:379-82.

3. Takeuchi S, Sugawara T, Masaoka H, Takasato Y. Fourth ventricular meningioma: A case report and literature review. Acta Neurol Belg 2012;112:97-100.

4. Carlotti CG Jr., Neder L, Colli BO, dos Santos MB, Garcia AS, Elias J Jr., et al. Clear cell meningioma of the fourth ventricle. Am J Surg Pathol 2003;27:131-5.

5. Burgan OT, Bahl A, Critcher V, Zaki HS, McMullan PJ, Sinha S. Clear cell meningioma of the fourth ventricle in a child: A case report and literature review. Pediatr Neurosurg 2010;46:462-5.

6. Epari S, Sharma MC, Sarkar C, Garg A, Gupta A, Mehta VS. Chordoid meningioma, an uncommon variant of meningioma: A clinicopathologic study of 12 cases. J Neurooncol 2006;78:263-9.

7. Lyngdoh BT, Giri PJ, Behari S, Banerji D, Chhabra DK, Jain VK. Intraventricular meningiomas: A surgical challenge. J Clin Neurosci 2007;14:442-8. 